\title{
Kein erhöhtes Risiko für stumme Hirninfarkte und White matter lesions bei Migräne mit Aura
}

Fragestellung: Haben Patienten, die eine Migräne mit Aura haben, ein höheres Risiko für klinisch stumme Hirninfarkte und „White matter lesions“?

Hintergrund: Viele epidemiologische Studien haben gezeigt, dass ein Zusammenhang zwischen der Migräne mit Aura und einem erhöhten Risiko von kardiovaskulären Erkrankungen, insbesondere von Schlaganfällen besteht. Vor einigen Jahren gab es eine aufsehenerregende Studie aus den Niederlanden, die mithilfe der Kernspintomografie zeigte, dass Patienten, die eine Migräne mit Aura haben, auch ein erhöhtes Risiko für klinisch stumme Hirninfarkte und vermehrte White matter lesions haben $[1,2,3]$. Dieses Ergebnis konnte allerdings in darauffolgenden kleineren Studien nicht repliziert werden. Die Arbeitsgruppe aus Dänemark hat sich jetzt vorgenommen, diese

Gaist D, Garde E, Blaabjerg M et al. Migraine with aura and risk of silent brain infarcts and white matter hyperintensities: an MRI study. Brain 2016; 139: $2015-23$
Fragestellung nochmals $\mathrm{zu}$ untersuchen.

Patienten und Methodik: Der erste Teil der Studienteilnehmer wurde aus dem däni- schen Zwillingsregister rekrutiert. Dabei wurden jeweils Zwillingspaare identifiziert, bei denen ein Zwilling eine Migräne mit Aura aufwies und der andere keine Migräne hatte. Bei allen Zwillingen wurden dann 3-Tesla-Kernspintomografien durchgeführt und verblindet ausgewertet. Dabei fanden sich 172 Migränepatienten, bei denen beide Zwillinge eine Migräne hatten, 34 Zwillinge ohne Migräne und 139 Kontrollen ohne Migräne.

Ergebnisse: Die Patienten waren im Mittel 48 Jahre alt und $40 \%$ waren monozygote Zwillinge. Vaskuläre Risikofaktoren waren bei Migränepatienten und Kontrollen gleich häufig. Stumme Hirninfarkte fanden sich viermal bei den Patienten mit Migräne und einmal bei den Kontrollen. Dieser Unterschied war nicht signifikant. Unter Verwendung verschiedener Scores ergab sich kein Unterschied in der Ausprägung und Häufigkeit von White matter lesions.

Schlussfolgerungen: In einer großen Zwillingsstudie in Dänemark ergab sich kein Hinweis auf ein erhöhtes Risiko für klinisch stumme Hirninfarkte oder White matter lesions bei Patienten, die eine Migräne mit Aura haben.

- Kommentar von Hans-Christoph Diener, Essen

\section{Leider eine Querschnitts- und keine Längsschnittstudie}

Die Ergebnisse dieser großen populationsbezogenen Zwillingsstudie weichen von der holländischen CAMERA-Studie $\mathrm{ab}$, in der sich bei Patienten, die unter einer Migräne mit Aura

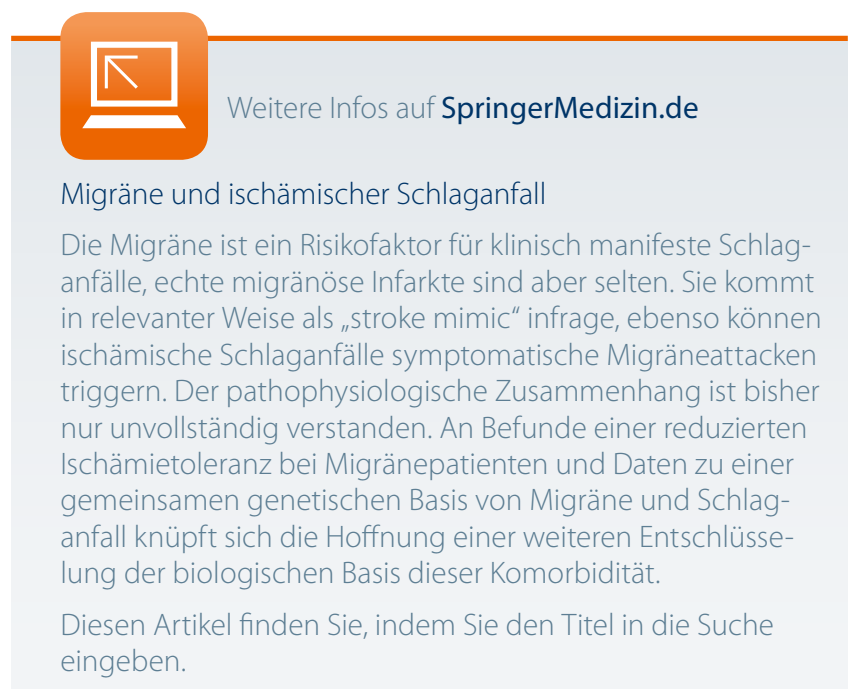

litten, sowohl ein erhöhtes Risiko von stummen Hirninfarkten als auch von White matter lesions fand. Die dänische Studie ist größer als die Studie aus den Niederlanden und die Verwendung von Zwillingspaaren verhindert eine unterschiedliche Prävalenz von vaskulären Risikofaktoren. Der einzige Nachteil der dänischen Studie ist, dass es sich um eine Querschnittsund nicht um eine Längsschnittstudie handelt.
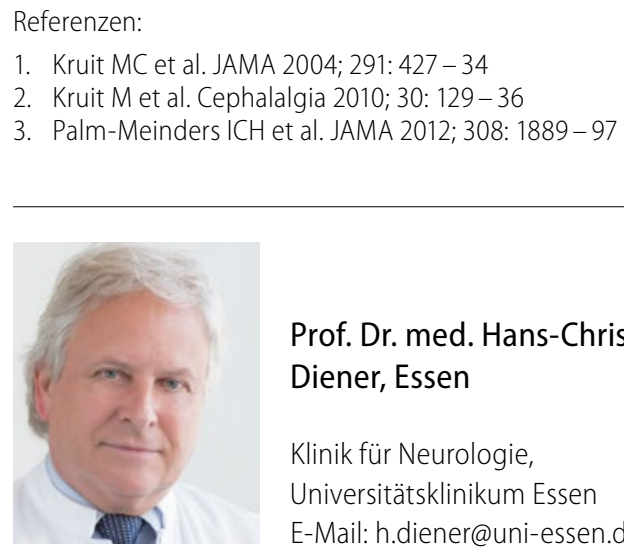

Prof. Dr. med. Hans-Christoph Diener, Essen

Klinik für Neurologie, Universitätsklinikum Essen E-Mail:h.diener@uni-essen.de 